\title{
New Approximation Algorithms for Minimum Cycle Bases of Graphs*
}

\author{
Telikepalli Kavitha $^{\dagger} \quad$ Kurt Mehlhorn $^{\ddagger} \quad$ Dimitrios Michail $^{\ddagger}$
}

\begin{abstract}
We consider the problem of computing an approximate minimum cycle basis of an undirected non-negative edge-weighted graph $G$ with $m$ edges and $n$ vertices; the extension to directed graphs is also discussed. In this problem, a $\{0,1\}$ incidence vector is associated with each cycle and the vector space over $\mathbb{F}_{2}$ generated by these vectors is the cycle space of $G$. A set of cycles is called a cycle basis of $G$ if it forms a basis for its cycle space. A cycle basis where the sum of the weights of the cycles is minimum is called a minimum cycle basis of $G$.

Cycle bases of low weight are useful in a number of contexts, e.g. the analysis of electrical networks, structural engineering, chemistry, and surface reconstruction. Although in most such applications any cycle basis can be used, a low weight cycle basis often translates to better performance and/or numerical stability. Despite the fact that the problem can be solved exactly in polynomial time, we design approximation algorithms since the performance of the exact algorithms may be too expensive for some practical applications.

We present two new algorithms to compute an approximate minimum cycle basis. For any integer $k \geq 1$, we give $(2 k-1)$-approximation algorithms with expected running time $O\left(k m n^{1+2 / k}+\right.$ $\left.m n^{(1+1 / k)(\omega-1)}\right)$ and deterministic running time $O\left(n^{3+2 / k}\right)$, respectively. Here $\omega$ is the best exponent of matrix multiplication. It is presently known that $\omega<2.376$. Both algorithms are $o\left(m^{\omega}\right)$ for dense graphs. This is the first time that any algorithm which computes sparse cycle bases with a guarantee drops below the $\Theta\left(m^{\omega}\right)$ bound.

We also present a 2-approximation algorithm with expected running time $O\left(m^{\omega} \sqrt{n \log n}\right)$, a linear time 2-approximation algorithm for planar graphs and an $O\left(n^{3}\right)$ time 2.42-approximation algorithm for the complete Euclidean graph in the plane.
\end{abstract}

\section{Introduction}

Let $G=(V, E)$ be an undirected connected graph with $m$ edges and $n$ vertices. A cycle of $G$ is any subgraph of $G$ where each vertex has even degree. Associated with each cycle $C$ is an incidence vector $x$, indexed on $E$, where for any $e \in E, x_{e}$ is 1 if $e$ is an edge of $C$ and 0 otherwise. The vector space over $\mathbb{F}_{2}$ generated by the incidence vectors of cycles is called the cycle space of $G$. It is well known that this vector space has dimension $N=m-n+1$, where $m$ is the number of edges of $G$ and $n$ is the number of vertices. A maximal set of linearly independent cycles is called a cycle basis. The edges of $G$ have

\footnotetext{
*A preliminary version of this paper appeared in the 24th International Symposium on Theoretical Aspects of Computer Science [20].

$\dagger$ Indian Institute of Science, Bangalore, India; kavitha@csa.iisc.ernet.in

‡Max-Planck-Institut für Informatik, Saarbrücken, Germany; \{mehlhorn,michail\}@ mpi-inf.mpg.de

${ }^{\S}$ Corresponding address: Dimitrios Michail, Max-Planck-Institut für Informatik, Stuhlsatzenhausweg 85, 66123 Saarbrücken, Germany. email: michail@mpi-inf.mpg.de
} 
non-negative weights assigned to them. A cycle basis where the sum of the weights of the cycles is minimum is called a minimum cycle basis of $G$. We use the abbreviation MCB to refer to a minimum cycle basis. Minimum cycle bases are of considerable practical importance and therefore the problem of computing an MCB has received considerable attention. An early paper is by Stepanec [29]. Horton [16] presented the first polynomial time algorithm. Faster and/or alternative algorithms were later presented by de Pina [10], Golynski and Horton [12], Berger et al. [4], and Kavitha et al. [21]. The current fastest algorithm [21] has running time $O\left(m^{2} n+m n^{2} \log n\right)$. Implementations are discussed in $[17,9,26]$.

One of the most important areas of application of the MCB problem is electrical networks [30, $10,4]$. Many problems arising in the design and analysis of electrical networks can be formulated in graph-theoretic terms. In fact, in the analysis of complex electrical networks by graph theoretical methods, a basic problem is to determine the solvability of the "network equation", a system of algebraic differential equations that describes the relation of currents and voltages in a network as functions of time. In order to check structural solvability of that system quickly by a heuristic matching approach, fast algorithms are needed that compute sparse representations. The equations corresponding to the Kirchhoff voltage law are critical, since for the remaining equations, a sparse representation is readily available. Hence the central problem is that of computing a sparse cycle basis to describe the "voltage law" part of the system.

Other applications are in structural engineering [6], chemistry and biochemistry [11, 23], and surface reconstruction from point clouds [31]. In most applications, the computation of an MCB is a preprocessing step. The use of an MCB ensures sparseness and translates into faster running times of the main algorithm. Unfortunately, even the fastest exact minimum cycle basis algorithm has a running time of $\Theta\left(m^{2} n+m n^{2} \log n\right)$. This may dominate the running time of the application.

However, most applications can work with any cycle basis and any constant factor approximate minimum cycle basis may be substituted for a minimum cycle basis without much affect on the application. In [21] an $\alpha$-approximation algorithm for any $\alpha>1$ is presented for the MCB problem; its running time is $o\left(m^{2} n+m n^{2} \log n\right)+\Theta\left(m^{\omega}\right)$, where $\omega$ is the exponent of matrix multiplication. It is known [7] that $\omega<2.376$. The time bound of $\Theta\left(m^{\omega}\right)$ is still prohibitive for some of the applications. It results from Gaussian elimination on $m \times m$ linear systems.

We present a new approximation approach which leads to vastly improved time bounds. In particular, for any integer $k \geq 1$ we give two $(2 k-1)$-approximation algorithms with expected running time $O\left(k m n^{1+2 / k}+m n^{(1+1 / k)(\omega-1)}\right)$ and deterministic running time $O\left(n^{3+2 / k}\right)$, respectively. Both algorithms are $o\left(m^{\omega}\right)$ for sufficiently dense graphs, the first algorithm for $m>\max \left(n^{1+1 / k}, \sqrt[\omega-1]{k n^{1+2 / k}}\right)$ and the second algorithm for $m>n^{\frac{3}{\omega}+\frac{2}{k \omega}}>n^{1.26+\frac{0.84}{k}}$. The first algorithm is faster for sparser and the second algorithm for denser graphs. More precisely, the second algorithm is faster for $m>n^{4-\omega+\frac{3-\omega}{k}}$ which with the current upper bound on $\omega$ is $m>n^{1.624+\frac{0.624}{k}}$.

Our algorithms work in two phases. The first phase is a very fast computation of a large number of cycles (all but $O\left(n^{1+1 / k}\right)$ cycles) in an approximate MCB. The second part is a more expensive computation of the remaining cycles. We present two different ways for computing these remaining cycles, leading to the above two algorithms, each faster for different graph densities. Only the second phase needs a null space computation; it is a null space computation of a square system of size $O\left(n^{1+1 / k}\right)$. Our new algorithms are fast even when implemented without fast matrix multiplication. Furthermore, by combining the techniques of both the algorithms, we get an even faster algorithm at the expense of a larger approximation factor.

We also present a 2-approximation algorithm with an expected running time of $O\left(m^{\omega} \sqrt{n \log n}\right)$. For sparse graphs, this is subcubic. Moreover, we develop very fast approximation algorithms for 
some special graph classes. For planar graphs we give a linear time 2-approximation algorithm and for the complete Euclidean graph in the plane we give a 2.42-approximation algorithm with running time $O\left(n^{3}\right)$. In higher dimensions we give a $k$-approximation algorithm for any $k>1$ with running time $O\left(s^{d} n^{3} \log n\right)$ where $s=4(k+1) /(k-1)$ and $d$ is the fixed dimension.

The minimum cycle basis problem for directed graphs is less studied. Polynomial time algorithms are given in $[19,24,13,18]$. The fastest deterministic algorithm [13] has running time $O\left(m^{3} n+\right.$ $\left.m^{2} n^{2} \log n\right)$ and there is a Monte Carlo algorithm [18, 13] with running time $O\left(m^{2} n+m n^{2} \log n\right)$. Some of our algorithms generalize to directed graphs. We give a deterministic $(2 k-1)$-approximation algorithm with running time $O\left(n^{4+3 / k}\right)$, a Monte Carlo $(2 k-1)$-approximation algorithm with running time $O\left(n^{3+2 / k}\right)$, and a 2-approximation algorithm with expected running time $O\left(m^{\omega} \sqrt{n \log n}\right)$.

The paper is organized as follows. Section 2 contains some necessary background and a simple lower bound for the weight of an MCB. Section 3 presents our approach for computing fast a large number of cycles of an approximate MCB. Section 4 contains two different algorithms in order to compute the remaining cycles. In these sections we also extend our techniques to directed (Section 4.4) and some special classes (Section 4.5) of graphs. Practical issues are discussed in Section 5. In Section 6 we present our 2-approximation algorithm. We conclude with open problems in Section 7.

\section{Preliminaries}

Let $T$ be any spanning tree in $G(V, E)$, let $e_{1}, \ldots, e_{N}$ be the edges of $E \backslash T$ in some arbitrary but fixed order, and let $e_{N+1}, \ldots, e_{m}$ be the edges in $T$ in some arbitrary but fixed order. We frequently view cycles in terms of their restricted incidence vectors ${ }^{1}$, that is, each cycle is a vector in $\{0,1\}^{N}$.

We use $S$ and $R$ to denote subsets of $E \backslash T$. Each such subset gives rise to an incidence vector in $\{0,1\}^{N}$. We use $\langle C, S\rangle$ to denote the standard inner product of vectors $C$ and $S$. We say that a vector $S$ is orthogonal to $C$ if $\langle C, S\rangle=0$. In the field $\mathbb{F}_{2},\langle C, S\rangle=1$ if and only if $C$ contains an odd number of edges of $S$.

For a cycle $C$, we use $w(C)=\sum_{e \in C} w(e)$ to denote its weight. We use $w_{G}(\mathrm{MCB})$ to denote the weight of a minimum cycle basis of graph $G$. When it is clear by the context we omit $G$ and write $w(\mathrm{MCB})$. The following lemma gives us a lower bound on $w(\mathrm{MCB})$. We include the proof given in [21].

Lemma 2.1 (de Pina [10]). Let $R_{1}, \ldots, R_{N}$ be linearly independent vectors in $\{0,1\}^{N}$ and let $A_{i}$ be a shortest cycle in $G$ such that $\left\langle A_{i}, R_{i}\right\rangle=1$. Then $\sum_{i=1}^{N} w\left(A_{i}\right) \leq w(\mathrm{MCB})$.

Proof. Let $C_{1}, \ldots, C_{N}$ be the cycles of an MCB. We may assume without loss of generality that the $A_{i}$ vectors and the $C_{i}$ vectors are sorted by weight, that is, $w\left(A_{1}\right) \leq w\left(A_{2}\right) \leq \ldots \leq w\left(A_{N}\right)$ and $w\left(C_{1}\right) \leq$ $w\left(C_{2}\right) \leq \ldots \leq w\left(C_{N}\right)$. The former may require a renumbering of the $R_{i}$ vectors. It suffices to show $w\left(A_{i}\right) \leq w\left(C_{i}\right)$ for all $i$.

Consider a fixed $i$. We have that $\left\langle C_{k}, R_{\ell}\right\rangle=1$ for some $k$ and $\ell$ with $1 \leq k \leq i \leq \ell \leq N$. Otherwise, the $N-i+1$ linearly independent vectors $R_{i}, R_{i+1}, \ldots, R_{N}$ belong to the subspace orthogonal

\footnotetext{
${ }^{1}$ For a cycle $C$, use $C$ to denote its incidence vector in $\{0,1\}^{N}$ and $C^{*}$ to denote its incidence vector in $\{0,1\}^{m}$. Consider a set of cycles $C_{1}, \ldots, C_{k}$. Clearly, if the vectors $C_{1}^{*}$ to $C_{k}^{*}$ are dependent, then so are the vectors $C_{1}$ to $C_{k}$. Conversely, assume that $\sum_{i} \lambda_{i} C_{i}=0$. Then $C=\sum_{i} \lambda_{i} C_{i}^{*}$ contains only edges in $T$. Moreover, since $C$ is a sum of cycles, each vertex has even degree with respect to $C$. Thus, $C=0$ and hence linear dependence of the restricted incidence vectors implies linear dependence of the full incidence vectors. For this reason, we may restrict attention to the restricted incidence vectors when discussing questions of linear independence.
} 
to $C_{1}, \ldots, C_{i}$; however, this subspace has dimension only $N-i$. Thus, $w\left(A_{\ell}\right) \leq w\left(C_{k}\right)$ since $A_{\ell}$ is a shortest cycle such that $\left\langle A_{\ell}, R_{\ell}\right\rangle=1$. But by the sorted order, $w\left(A_{i}\right) \leq w\left(A_{\ell}\right)$ and $w\left(C_{k}\right) \leq w\left(C_{i}\right)$. This implies $w\left(A_{i}\right) \leq w\left(C_{i}\right)$.

The sets $R_{i}=\left\{e_{i}\right\}, 1 \leq i \leq N$, are clearly independent. The shortest cycle $C$ with $\left\langle C, R_{i}\right\rangle=1$ consists of the edge $e_{i}$ plus the shortest path in $G \backslash\left\{e_{i}\right\}$ connecting its endpoints. We use $S C_{i}$ to denote this cycle. The cycle exists, since there is always the spanning tree path in $E \backslash\left\{e_{i}\right\}$ connecting the endpoints of $e_{i}$. Let $\mathrm{SC}=\left\{S C_{i} \mid 1 \leq i \leq N\right\}$ be the shortest cycle multiset and let $w(\mathrm{SC})=\sum_{C \in \mathrm{SC}} w(C)$ be its weight. Thus, we obtain Corollary 2.2.

Corollary 2.2. $w(\mathrm{SC}) \leq w(\mathrm{MCB})$.

\section{The new approach}

Our approximation algorithms are motivated by the shortest cycle multiset lower bound (Corollary 2.2). We fix a parameter $\lambda \leq N$ and construct a set of linearly independent cycles $C_{1}, \ldots, C_{\lambda}$ such that $w\left(C_{i}\right) \leq(2 k-1) \cdot w\left(S C_{i}\right)$ for $1 \leq i \leq \lambda$. In the second phase, we extend the partial basis to a full basis. We offer two alternatives for the second phase. Let $t=2 k-1$.

Now we give the details of the first phase. We construct the cycles $C_{1}, \ldots, C_{\lambda}$ using a sparse $t$ spanner of $G$. A multiplicative $t$-spanner of a graph $G$ is a subgraph $G^{\prime}\left(V, E^{\prime}\right), E^{\prime} \subseteq E$ such that for any $u, v \in V$ we have $w\left(\operatorname{SP}_{G^{\prime}}(u, v)\right) \leq t \cdot w\left(\operatorname{SP}_{G}(u, v)\right)$ where $\operatorname{SP}_{G}(u, v)$ denotes a shortest path in $G$ from $u$ to $v$. When it is clear from the context we omit the subscript $G$ and write $\operatorname{SP}(u, v)$. Let $G^{\prime}\left(V, E^{\prime}\right)$ be such a $t$-spanner of $G$. Since we can always add the edges in $T$ to $E^{\prime}$ (recall that $T$ is an arbitrary spanning tree of $G$ ), we may assume $T \subseteq E^{\prime}$. We also assume that the edges are indexed such that $E \backslash E^{\prime}=\left\{e_{1}, \ldots, e_{\lambda}\right\}$.

For each edge $e_{i}=(u, v) \in E \backslash E^{\prime}$, let $C_{i}$ be formed by $e_{i}$ and $\mathrm{SP}_{G^{\prime}}(u, v)$. The cycles $C_{i}, 1 \leq i \leq \lambda$ are clearly independent since $e_{i}$ is contained in precisely $C_{i}$. We have $w\left(C_{i}\right)=w\left(e_{i}\right)+w\left(\operatorname{SP}_{G^{\prime}}(u, v)\right) \leq$ $w\left(e_{i}\right)+t \cdot w\left(\mathrm{SP}_{G}(u, v)\right) \leq t \cdot w\left(S C_{i}\right)$.

The running time of phase 1 is easily estimated. As pointed out by Althöfer et al. [1] every weighted undirected graph on $n$ vertices has a $(2 k-1)$-spanner with $O\left(n^{1+1 / k}\right)$ edges where $k \geq 1$ is an integer. Such a spanner can be constructed using an algorithm similar to Kruskal's algorithm (see Cormen et al. [8]) for constructing minimum spanning trees. In order to build the spanner, consider all edges of the graph in non-decreasing order of weight, adding each edge to the spanner if its endpoints are not already connected, in the spanner, by a path using at most $2 k-1$ edges. At any stage, the spanner is a $(2 k-1)$-spanner of the edges already considered, and its unweighted girth is at least $2 k+1$, so it has only $O\left(n^{1+1 / k}\right)$ edges. The above procedure can be implemented in $O\left(m n^{1+1 / k}\right)$ time.

In the above spanner we are going to perform $\lambda$ shortest path computations, one for each edge of $G$ that is not in the spanner. Using Dijkstra's algorithm we need time $O\left(\lambda \cdot\left(n^{1+1 / k}+n \log n\right)\right)$ and since $\lambda \leq m$ we can compute both the spanner and the $\lambda$ linearly independent cycles in time $O\left(m n^{1+1 / k}\right)$. We should mention that there are faster algorithms to construct similar spanners, see for example [33]. However, the construction by Althöfer et al. suffices for our purposes.

\section{The remaining cycles}

In the preceding section we computed most of the cycles of an approximate minimum cycle basis. We are left with computing the remaining cycles. The number of additional cycles is $N-\lambda$. Note that this is exactly the dimension of the cycle space of the spanner $G^{\prime}$. We present two different algorithms. 


$$
\begin{array}{ll}
\text { input } & : \text { Graph } G(V, E) \text { and integer } k \geq 1 . \\
\text { output } & : \mathrm{A}(2 k-1) \text {-approximate } \mathrm{MCB} .
\end{array}
$$

Construct a $(2 k-1)$-spanner $G^{\prime}$ with $O\left(n^{1+1 / k}\right)$ edges. Let $e_{1}, \ldots, e_{\lambda}$ be the edges of $G \backslash G^{\prime}$. For $1 \leq i \leq \lambda$ let $C_{i}=e_{i}+p_{i}$ where $e_{i}=\left(u_{i}, v_{i}\right)$ and $p_{i}$ is a shortest path in $G^{\prime}$ from $u_{i}$ to $v_{i}$. Find linearly independent $S_{\lambda+1}, \ldots, S_{N}$ in the subspace orthogonal to cycles $C_{1}, \ldots, C_{\lambda}$.

Call the algorithm in [21] with input: the graph $G$, sets $\left\{C_{1}, \ldots, C_{\lambda}\right\},\left\{S_{\lambda+1}, \ldots, S_{N}\right\}$ and $N-\lambda$ to compute $(2 k-1)$-approximate cycles $C_{\lambda+1}, \ldots, C_{N}$.

Return $\left\{C_{1}, \ldots, C_{\lambda}\right\} \cup\left\{C_{\lambda+1}, \ldots, C_{N}\right\}$.

Figure 1: The first approximation algorithm.

The first approach uses all the edges in $G$ to construct the remaining cycles while the second approach uses only the edges $e_{\lambda+1}, \ldots, e_{m}$.

\subsection{The first approach}

We first need to briefly review the algorithm in [21] in order to compute a minimum cycle basis; it refines a previous algorithm by de Pina [10]. The algorithm is recursive. We immediately describe the modification of the algorithm needed for our purposes.

The general step adds some number $k$ of cycles to a partial basis PB of size $\alpha$. This step takes as input an integer $k \geq 1$, and $k$ linearly independent vectors $S_{\alpha+1}, \ldots, S_{\alpha+k}$ orthogonal to the cycles in PB. The vectors, when viewed as sets, have the additional property that $S_{\alpha+i} \cap\left\{e_{\alpha+1}, \ldots, e_{N}\right\}=$ $\left\{e_{\alpha+i}\right\}$ for $1 \leq i \leq k$. The step updates the sets $S_{\alpha+1}, \ldots, S_{\alpha+k}$ and returns $k$ cycles $Z_{\alpha+1}, \ldots, Z_{\alpha+k}$ such that $\left\langle Z_{i}, S_{j}\right\rangle=\delta_{i j}$ for $\alpha+1 \leq i \leq j \leq \alpha+k$ (here $\delta_{i j}$ is 1 if $i=j$ and 0 otherwise). The update has the additional property that it does not affect the orthogonality with respect to the partial basis PB. Observe, that the cycles $\mathrm{PB} \cup\left\{Z_{\alpha+1}, \ldots, Z_{\alpha+k}\right\}$ are linearly independent. To see this note that for any $1 \leq i \leq k,\left\langle Z_{\alpha+i}, S_{\alpha+i}\right\rangle=1$ while any cycle $C$ in the span of $\mathrm{PB} \cup\left\{Z_{\alpha+1}, \ldots, Z_{\alpha+i-1}\right\}$ has $\left\langle C, S_{\alpha+i}\right\rangle=0$.

The top level call: We call the recursive procedure with the partial basis of phase 1 , namely $\mathrm{PB}=$ $\left\{C_{1}, \ldots, C_{\lambda}\right\}$ and ask it to compute $\mu=N-\lambda$ additional cycles. Let us write the $C_{1}, \ldots, C_{\lambda}$ in the form of a $\lambda \times N$ matrix with one row per cycle. Then

$$
\left(\begin{array}{c}
C_{1} \\
\vdots \\
C_{\lambda}
\end{array}\right)=\left(\begin{array}{ll}
I_{\lambda} & B
\end{array}\right)
$$

where $I_{\lambda}$ is the $\lambda \times \lambda$ identity matrix and $B$ is a $\lambda \times \mu$ matrix. The matrix has this form since each of the edges $e_{i}$ for $1 \leq i \leq \lambda$ belongs only to the cycle $C_{i}$. Set

$$
\left(\begin{array}{lll}
S_{\lambda+1} & \ldots & S_{\lambda+\mu}
\end{array}\right)=\left(\begin{array}{c}
B \\
I_{\mu}
\end{array}\right) .
$$

Then the product of the matrix of $C$ 's on the left side of Equation (1) and the matrix of $S$ 's on the left side of Equation (2) is $B+B=0$, i.e., the $S$ 's are orthogonal to the $C$ 's. Moreover, $S_{\lambda+i} \cap$ 
$\left\{e_{\lambda+1}, \ldots, e_{N}\right\}=\left\{e_{\lambda+i}\right\}$ for $1 \leq i \leq \mu$. The running time required to compute this null space basis is the time required to output the already known matrix $B$. By using some sparse representation of the vectors we need at most $O(\lambda \cdot \mu)$ time. In the general case $\lambda \leq m$ and $\mu=N-\lambda \in O\left(n^{1+1 / k}\right)$. Thus, initialization of phase 2 needs $O\left(m n^{1+1 / k}\right)$ time.

The recursive case, $k \geq 2$ : Let $\ell=\lceil k / 2\rceil$. We first call the algorithm recursively with $\ell$ and $S_{\alpha+1}$ to $S_{\alpha+\ell}$. The call will return cycles $Z_{\alpha+1}$ to $Z_{\alpha+\ell}$ and updated sets $S_{\alpha+1}$ to $S_{\alpha+\ell}$. We next update the sets $S_{\alpha+\ell+1}$ to $S_{\alpha+k}$. The set $S_{\alpha+j}, \ell+1 \leq j \leq k$, is replaced by a sum $S_{\alpha+j}+\sum_{1 \leq i \leq \ell} \beta_{j i} S_{\alpha+i}$ where the $\beta_{j i}$ are chosen such that the updated $S_{\alpha+j}$ becomes orthogonal to the cycles $Z_{\alpha+1}$ to $Z_{\alpha+\ell}$. Observe that orthogonality to the cycles in PB is not affected. The computation of the $\beta_{j i}$ 's and the update step can be implemented using fast matrix multiplication and takes time $O\left(m k^{\omega-1}\right)$ (see [21] for the details). The final step is to call the algorithm recursively for the remaining cycles. We therefore have the following recursion for the running time: $T(k)=T(\lceil k / 2\rceil)+T(\lfloor k / 2\rfloor)+O\left(m k^{\omega-1}\right)$ for $k \geq 2$. This solves to $T(k)=k \cdot T(1)+O\left(m k^{\omega-1}\right)$. We call the algorithm with $k=\mu$ and hence have total running time $\mu \cdot T(1)+O\left(m \mu^{\omega-1}\right)$.

The base case, $k=1$ : The algorithm computes a $t$-approximate shortest ${ }^{2}$ cycle $C$ with $\left\langle C, S_{\alpha+1}\right\rangle=1$. The shortest cycle $C$ with $\left\langle C, S_{\alpha+1}\right\rangle=1$ can be computed as follows. We set up an auxiliary graph $G^{\dagger}$ with two copies, say $v^{\prime}$ and $v^{\prime \prime}$, for each vertex $v$, and two copies $e^{\prime}$ and $e^{\prime \prime}$ for each edge $e=(u, v) \in E$. If $e \in S_{\alpha+1}$, the copies are $\left(u^{\prime}, v^{\prime \prime}\right)$ and $\left(u^{\prime \prime}, v^{\prime}\right)$ and if $e \notin S_{\alpha+1}$, the copies are $\left(u^{\prime}, v^{\prime}\right)$ and $\left(u^{\prime \prime}, v^{\prime \prime}\right)$. Then a shortest cycle $C$ with $\left\langle C, S_{\alpha+1}\right\rangle=1$ corresponds to a shortest path connecting the two copies of some vertex $v$ minimized over all $v$. Such a path can be found by $n$ shortest path computations in the auxiliary graph. In order to compute a $t$-approximate shortest cycle $C$ with $\left\langle C, S_{\alpha+1}\right\rangle=1$ we compute $t$-approximate single source shortest paths between $n$ pairs of vertices.

We need to perform a total of $\mu n$ approximate shortest path computations. Therefore, we require a faster algorithm than constructing a spanner. We use an approximate distance oracle. Thorup and Zwick [33] constructed a structure which answers $(2 k-1)$-approximate shortest path queries in time $O(k)$. The structure requires space $O\left(k n^{1+1 / k}\right)$ and can be constructed in expected time $O\left(k m n^{1 / k}\right)$. In the case of unweighted graphs Baswana and Sen [3] showed that a $(2 k-1)$-approximate distance oracle can be computed in expected $O\left(\min \left(n^{2}, k m n^{1 / k}\right)\right)$ time. The same can also be done deterministically [28].

Using such a construction, we bound $T(1)$ by the cost of computing the approximate distance oracle $\left(O\left(k m n^{1 / k}\right)\right.$ expected time) and the cost of performing $n$ queries to the oracle. Each query costs $O(k)$ and thus a total cost of $O(n k)$. Forming the actual cycle can be done in time linear in its length which is $O(n)$. Thus, $T(1)=O\left(k m n^{1 / k}\right)$ and therefore $T(\mu)=O\left(\mu k m n^{1 / k}+m \mu^{\omega-1}\right)$. Since $\mu \in O\left(n^{1+1 / k}\right)$ we get a bound of $O\left(k m n^{1+2 / k}+m n^{(1+1 / k)(\omega-1)}\right)$.

Approximation guarantee. We prove that the computed set of cycles is a $t$-approximation of the MCB. Consider the vectors $S_{\lambda+1}, \ldots, S_{N}$ at the end of the algorithm and define $S_{i}=\left\{e_{i}\right\}$ for $1 \leq i \leq \lambda$. Then each $C_{i}, 1 \leq i \leq N$, is a $t$-approximation of the shortest cycle in $G$ having odd intersection with $S_{i}$. All we need to show is Lemma 4.1. Then the approximation guarantee follows from Lemma 2.1.

Lemma 4.1. The vectors $S_{1}, \ldots, S_{N}$ are linearly independent.

Proof. Consider any $i$. We have $\left\langle C_{i}, S_{i}\right\rangle=1$ and $\left\langle C_{i}, S_{j}\right\rangle=0$ for all $j \geq i+1$. The latter holds for $j>\lambda$ by the invariants of the recursive procedure and it holds for $i<j \leq \lambda$ since $C_{i}$ consists of edge

\footnotetext{
${ }^{2}$ The original algorithm constructs a shortest cycle.
} 


$$
\begin{array}{ll}
\text { input } & : \text { Graph } G(V, E) \text { and integer } k \geq 1 . \\
\text { output } & : \mathrm{A}(2 k-1) \text {-approximate } \mathrm{MCB} .
\end{array}
$$

Construct a $(2 k-1)$-spanner $G^{\prime}$ with $O\left(n^{1+1 / k}\right)$ edges. Let $e_{1}, \ldots, e_{\lambda}$ be the edges of $G \backslash G^{\prime}$. For $1 \leq i \leq \lambda$ let $C_{i}=e_{i}+p_{i}$ where $e_{i}=\left(u_{i}, v_{i}\right)$ and $p_{i}$ is the shortest path in $G^{\prime}$ from $u_{i}$ to $v_{i}$.

Call the exact algorithm in [21] to find an MCB of $G^{\prime}$. Let these cycles be $C_{\lambda+1}, \ldots, C_{N}$. Return $\left\{C_{1}, \ldots, C_{\lambda}\right\} \cup\left\{C_{\lambda+1}, \ldots, C_{N}\right\}$.

Figure 2: The second approximation algorithm.

$e_{i}$ and edges in the spanner (which have index greater than $\lambda$ ) and $S_{j}=\left\{e_{j}\right\}$. Thus, $S_{i}$ is independent of the $S_{i+1}, \ldots, S_{N}$ and the lemma follows.

Theorem 4.2. $A(2 k-1)$-approximate minimum cycle basis, for any integer $k \geq 1$, in an undirected weighted graph can be computed in expected time $O\left(k m n^{1+2 / k}+m n^{(1+1 / k)(\omega-1)}\right)$.

The above theorem implies that an $O(\log n)$-approximate minimum cycle basis can be computed in expected time $O\left(m n^{\omega-1}+m n \log n\right)$. Note that Rizzi [27] independently designed an $O(m n)$ time algorithm which computes an $O(\log n)$ approximate minimum cycle basis.

\subsection{The second approach}

Our second algorithm just computes a minimum cycle basis of the $t$-spanner $G^{\prime}$. The dimension of the cycle space of $G^{\prime}$ is $\mu=N-\lambda$ and thus we have the right number of cycles. Let $C_{\lambda+1}, \ldots, C_{N}$ be an MCB of $G^{\prime}$. Cycles $\left\{C_{1}, \ldots, C_{\lambda}\right\} \cup\left\{C_{\lambda+1}, \ldots, C_{N}\right\}$ are by definition linearly independent and we are also going to prove that they form a $t$-approximation of an MCB of $G$.

For $1 \leq i \leq \lambda$, we have $C_{i}=e_{i}+p_{i}$, where $p_{i}$ is a shortest path in $G^{\prime}$ between the endpoints of $e_{i}$. In order to show that cycles $C_{1}, \ldots, C_{N}$ are a $t$-approximation of the MCB, we again define appropriate linearly independent vectors $S_{1}, \ldots, S_{N} \in\{0,1\}^{N}$ and use Lemma 2.1. Consider the exact algorithm in [21] executing with the $t$-spanner $G^{\prime}$ as its input. Other than the cycles $C_{\lambda+1}, \ldots, C_{N}$, the algorithm also returns the vectors $R_{\lambda+1}, \ldots, R_{N} \in\{0,1\}^{N-\lambda}$ such that $\left\langle C_{i}, R_{j}\right\rangle=0$ for $\lambda+1 \leq i<j \leq N$ and $C_{i}$ is a shortest cycle in $G^{\prime}$ such that $\left\langle C_{i}, R_{i}\right\rangle=1$ for $\lambda+1 \leq i \leq N$. Moreover, the $(N-\lambda) \times(N-\lambda)$ matrix whose $j$-th row is $R_{j}$ is lower triangular with 1 in its diagonal. This implies that the $R_{j}$ 's are linearly independent. Given any vector $S \in\{0,1\}^{N}$ let $\tilde{S}$ be the projection of $S$ onto its last $N-\lambda$ coordinates. In other words, if $S$ is an edge set of $G$, then let $\tilde{S}$ be the edge set restricted only to the edges of $G^{\prime}$. We define $S_{j}$ for $1 \leq j \leq N$ as follows. Let $S_{1}, \ldots, S_{\lambda}$ be the first $\lambda$ unit vectors of $\{0,1\}^{N}$. For $\lambda+1 \leq j \leq N$ define $S_{j}$ as:

$$
S_{j}=\left(-\left\langle\tilde{C}_{1}, R_{j}\right\rangle, \ldots,-\left\langle\tilde{C}_{\lambda}, R_{j}\right\rangle, R_{j, 1}, R_{j, 2}, \ldots, R_{j,(N-\lambda)}\right),
$$

where $R_{j, 1}, \ldots, R_{j,(N-\lambda)}$ are the coordinates of the vector $R_{j} \in\{0,1\}^{N-\lambda}$. Note that the vectors $S_{j}$ for $1 \leq j \leq N$, defined above, are linearly independent. This is because the $N \times N$ matrix whose $j$-th row is $S_{j}$ is lower triangular with 1's in its diagonal. The above definition of $S_{j}$ 's is motivated by the property that for each $1 \leq i \leq \lambda$, we have $\left\langle C_{i}, S_{j}\right\rangle=-\left\langle\tilde{C}_{i}, R_{j}\right\rangle+\left\langle\tilde{C}_{i}, R_{j}\right\rangle=0$, since the cycle $C_{i}$ has 0 in all first $\lambda$ coordinates, except the $i$-th coordinate, which is 1 . Lemma 4.3, shown below, together with Lemma 2.1, implies the correctness of our approach. 
Lemma 4.3. Consider the above defined $S_{j}$ for $1 \leq j \leq N$ and let $D_{j}$ be a shortest cycle in $G$ such that $\left\langle D_{j}, S_{j}\right\rangle=1$. Cycle $C_{j}$ returned by the algorithm in Figure 2 has weight at most times the weight of $D_{j}$.

Proof. This is obvious for $1 \leq j \leq \lambda$ since $D_{j}$ is a shortest cycle in $G$ which uses edge $e_{j}$ and $C_{j}=$ $e_{j}+p_{j}$, where $p_{j}$ is a $t$-approximate shortest path between the endpoints of $e_{j}$. Consider now $D_{j}$ for $\lambda+1 \leq j \leq N$. If $D_{j}$ uses any edge $e_{i}$ for $1 \leq i \leq \lambda$ we replace it with the corresponding shortest path in the spanner. This is the same as saying consider the cycle $D_{j}+C_{i}$ instead of $D_{j}$. Let $D_{j}^{\prime}=$ $D_{j}+\sum_{1 \leq i \leq \lambda}\left(e_{i} \in D_{j}\right) C_{i}$ where $\left(e_{i} \in D_{j}\right)$ is 1 if $e_{i} \in D_{j}$ and 0 if $e_{i} \notin D_{j}$. Then

$$
\left\langle D_{j}^{\prime}, S_{j}\right\rangle=\left\langle D_{j}, S_{j}\right\rangle+\sum_{1 \leq i \leq \lambda}\left(e_{i} \in D_{j}\right)\left\langle C_{i}, S_{j}\right\rangle
$$

But recall that our definition of $S_{j}$ ensures that $\left\langle C_{i}, S_{j}\right\rangle=0$ for $1 \leq i \leq \lambda$. This implies that $\left\langle D_{j}^{\prime}, S_{j}\right\rangle=$ $\left\langle D_{j}, S_{j}\right\rangle=1$. But $D_{j}^{\prime}$ by definition has 0 in the first $\lambda$ coordinates and $\tilde{S}_{j}=R_{j}$, which in turn implies that

$$
\left\langle\tilde{D}_{j}^{\prime}, R_{j}\right\rangle=\left\langle\tilde{D}_{j}^{\prime}, \tilde{S}_{j}\right\rangle=\left\langle D_{j}^{\prime}, S_{j}\right\rangle=1 .
$$

$C_{j}$ is a shortest cycle in $G^{\prime}$ such that $\left\langle C_{j}, R_{j}\right\rangle=1$. Thus, $C_{j}$ has weight at most the weight of $\tilde{D}_{j}^{\prime}$ (which is the same cycle as $D_{j}^{\prime}$ ), and by construction, $D_{j}^{\prime}$ has weight at most $t$ times the weight of $D_{j}$.

Thus, we have shown that the cost of our approximate basis is at most $t$ times the cost of an optimal basis. As a $t$-spanner we will again use a $(2 k-1)$-spanner. The best time bound in order to compute an MCB is $O\left(m^{2} n+m n^{2} \log n\right)$ and since a $(2 k-1)$-spanner has at most $O\left(n^{1+1 / k}\right)$ edges the total running time becomes $O\left(n^{3+2 / k}\right)$. It is also known that any graph has an $O(\log n)$-spanner of linear size.

Theorem 4.4. A $(2 k-1)$-approximate minimum cycle basis, for any integer $k \geq 1$, in a weighted undirected graph can be computed in time $O\left(n^{3+2 / k}+n^{3+1 / k} \log n\right)$.

\subsection{Combining the approaches}

Consider the algorithm in Figure 2. After computing the first $\lambda$ cycles we use an exact MCB algorithm to compute the remaining cycles. We can instead use the algorithm in Figure 1 which is fast for sparse graphs. We begin by computing a $(2 k-1)$-spanner $G^{\prime}$ of $G$ and the first $\lambda$ cycles. Then we call the algorithm in Figure 1 to compute a $(2 q-1)$-approximate MCB of $G^{\prime}$. The result will be a $(2 k-1)(2 q-1)$-approximate MCB of $G$. Note that in this case it is not sufficient to use the $O\left(m n^{1+1 / k}\right)$ approach of Althöfer et al. [1] in order to compute the initial $(2 k-1)$-spanner $G^{\prime}$. Thorup and Zwick [33] have shown how to construct a $(2 k-1)$-spanner $G^{\prime}$ with size $\tilde{O}\left(k n^{1+1 / k}\right)$ in expected time $\tilde{O}\left(k m n^{1 / k}\right)$. Such a spanner is a collection of shortest path trees $T(w)$ for $w \in V$ and given two vertices $u, v \in V$ there is a tree in this collection that contains a path between $u$ and $v$ that is of stretch at most $2 k-1$. Furthermore, the corresponding tree can be obtained in $O(k)$ time. We can also preprocess these trees in order to be able to output the path in amortized or worst case constant time per edge.

Consider running the algorithm in Figure 1 in the subgraph $G^{\prime}$. The algorithm will first compute a $(2 q-1)$-spanner $G^{\prime \prime}$ of the $(2 k-1)$-spanner $G^{\prime}$ and some of the cycles. Then it will compute the remaining cycles by doing $(2 q-1)$-approximate shortest cycles computations in $G^{\prime}$. We therefore get an algorithm with expected running time $\tilde{O}\left(q k \cdot n^{1+1 / k} n^{1+2 / q}+k n^{1+1 / k} n^{(1+1 / q)(\omega-1)}\right)$. Note that this upper bound does not include the time to output the initial cycles using the $(2 k-1)$-spanner. 
Theorem 4.5. A $(2 k-1)(2 q-1)$-approximate MCB of an undirected weighted graph, for any two integers $k, q \geq 1$, can be computed in expected time $\tilde{O}\left(q k \cdot n^{2+1 / k+1 / q}\left(n^{1 / q}+n^{(1+1 / q)(\omega-2)}\right)\right)$ plus the time to output the MCB.

\subsection{Directed graphs}

Our techniques for computing a $2 k-1$ approximate minimum cycle basis can also be applied to the minimum cycle basis problem in directed graphs. A cycle in a directed graph is a cycle in the underlying undirected graph with edges traversable in both directions. A $\{-1,0,1\}$ edge incidence vector is associated with each cycle: edges traversed by the cycle in the right direction get 1 and edges traversed in the opposite direction get -1 . The cycle space is the space generated by these cycle vectors over $\mathbb{Q}$. Note that the weight of a cycle is simply the sum of the weight of its edges, independent of the orientation of these edges.

The algorithms for finding an MCB in directed graphs are based on the techniques used in [10,21]. Some more ideas are required in order to compensate for the extra cost of arithmetic that arises when changing the base field from $\mathbb{F}_{2}$ to $\mathbb{Q}$. Lemma 2.1 can also be generalized, see for example [19]. The algorithm in Figure 2 can be directly generalized. For the spanner computation we view our directed graph $G$ as undirected and we compute a $(2 k-1)$-spanner $G^{\prime}$. We then give to the edges of $G^{\prime}$ the orientation that they have in $G$.

As in Section 4.2 we return two sets of cycles. The first set is constructed as follows. For each edge $e_{i} \in E \backslash E^{\prime}$ for $1 \leq i \leq \lambda$ we compute the cycle $e_{i}+p_{i}$ where $p_{i}$ is the shortest path in $G^{\prime}$ between the endpoints of $e_{i}$ when $G^{\prime}$ is viewed as an undirected graph. Then, we traverse each such cycle in an arbitrary orientation and form our directed cycles based on the direction of the edges in $G$. The second set is simply the set of cycles of a directed MCB of $G^{\prime}$. The proof of correctness of this approach follows by Section 4.2 if we replace the base field with $\mathbb{Q}$.

The time to compute the spanner is again $O\left(m n^{1+1 / k}\right)$. The fastest deterministic algorithm [13] to compute an MCB of a directed graph has a running time of $\tilde{O}\left(m^{2} N^{\omega-1}+N^{3}\right)+O\left(N\left(m^{2} n+\right.\right.$ $\left.m n^{2} \log n\right)$ ). We execute this algorithm on the spanner and $G^{\prime}$ has at most $O\left(n^{1+1 / k}\right)$ edges. Thus, $N \leq m \in O\left(n^{1+1 / k}\right)$ and the running time becomes $O\left(m n^{1+1 / k}\right)+\tilde{O}\left(n^{(1+1 / k)(\omega+1)}\right)+O\left(n^{4+3 / k}\right)$. For $k \geq 1$ this is $O\left(n^{4+3 / k}\right)$.

Theorem 4.6. $A(2 k-1)$-approximate minimum cycle basis, for any integer $k \geq 1$, in a weighted directed graph can be computed in time $O\left(n^{4+3 / k}\right)$.

If we allow the use of randomization then we can compute approximate directed MCB even faster. The improved Monte Carlo algorithm in [13] computes an MCB of a directed graph with non-negative weights in time $O\left(m^{2} n+m n^{2} \log n\right)$ with probability at least $3 / 4$. Using this algorithm we get the following theorem.

Theorem 4.7. $A(2 k-1)$-approximate minimum cycle basis, for any integer $k \geq 1$, in a weighted directed graph can be computed with probability at least $3 / 4$ in time $O\left(n^{3+2 / k}\right)$.

\subsection{Planar and Euclidean graphs}

In the minimum cycle basis problem, some types of graphs are much easier to handle than others. Consider a planar graph $G$. In linear time we can find an embedding of $G$ in the plane. The approximate cycle basis algorithm just returns the set of bounded faces of $G$. It is easy to see that all these cycles are linearly independent and due to Euler's formula they are also the right number, $N=m-n+1=f-1$, where $f$ is the number of faces of $G$. 
For the approximation factor let $E^{*} \subseteq E$ be the edges of $G$ which belong to at least one cycle. Since each edge is incident with at most two faces this cycle basis has weight at most $2 \cdot \sum_{e \in E^{*}} w(e) \leq$ $2 \cdot w(\mathrm{MCB})$ since a minimum cycle basis has to use each edge that belongs to at least one cycle.

The embedding can be found in linear time [15]. The bounded faces can also be enumerated in linear time and the size of the output is also linear. Thus, the algorithm constructs in a trivial way a 2-approximate minimum cycle basis of a planar graph. We should also mention that Liebchen and Rizzi [25] present an algorithm which computes a minimum 2-basis of a planar graph (a basis in which each edge is contained in at most 2 cycles). Their algorithm, while more complicated, runs also in linear time and a minimum 2-basis is also a 2 approximation of the minimum cycle basis.

Let $P$ be a set of points in the Euclidean plane. The Euclidean graph $G(P)$ on $P$ has vertices corresponding to points in $P$. The graph is complete and the weight of an edge is the Euclidean distance between the points corresponding to the vertices. In order to find an approximate MCB of $G(P)$ we use the result that the Delaunay triangulation of the points $P$ is a $\approx 2.42$ [22] spanner of $G(P)$. Let DT denote the Delaunay triangulation of $P$. DT is a planar graph. The approximate MCB algorithm returns the following set of cycles: (a) For each edge $e=(u, v) \in G(P) \backslash$ DT the cycle $C_{e}$ formed by edge $e$ and the shortest path in DT from $u$ to $v$, and (b) a minimum cycle basis of DT.

Theorem 4.8. A 2.42 approximation of the MCB in a complete Euclidean graph in the plane can be constructed in $O\left(n^{3}\right)$ time.

Proof. The proof of the approximation ratio follows exactly the proof in Section 4.2. For the running time we note that computing the Delaunay triangulation requires $O(n \log n)$ time. The shortest path computations on the planar spanner require $O(\lambda n)$ which is $O\left(n^{3}\right)$. Computing an MCB of a planar graph can be done in $O\left(n^{2} \log n\right)$ by the algorithm of Hartvigsen and Mardon [14].

In the case we want to handle higher (but fixed) dimensions we can use the fact that the wellseparated pair decomposition [5] ( $s$-WSPD) for separation factor $s=4(t+1) /(t-1)$ is a $t$-spanner of $G(P)$ for $t>1$. The algorithm begins by computing an $s$-WSPD decomposition. The time to compute an $s$-WSPD is $O\left(n \log n+s^{d} n\right)$ where $d$ is the dimension. Since the size of the WSPD is $O\left(s^{d} n\right)$ we know that $\lambda \leq m$ and $\mu=N-\lambda \in O\left(s^{d} n\right)$. Thus, the time to compute the first $\lambda$ cycles is $O\left(m\left(s^{d} n+n \log n\right)\right)$.

For the remaining cycles we use the approach used by the algorithm in Figure 2. Computing exactly the MCB of the spanner we get a running time of $O\left(s^{d} n^{3} \log n\right)$. We have shown the following.

Theorem 4.9. A t-approximate $M C B$ for $t>1$ of a complete Euclidean graph can be computed in time $O\left(s^{d} n^{3} \log n\right)$ where $s=4(t+1) /(t-1)$ and $d$ the fixed dimension.

\section{Practical considerations}

The algorithms in Figure 1 and 2 use fast matrix multiplication. However, they are also efficient even when used without fast matrix multiplication. This fact has high practical value since high performance fast matrix multiplication libraries are difficult to implement. In the algorithm of Figure 2, instead of the $O\left(m^{2} n+m n^{2} \log n\right)$ algorithm to compute an MCB in $G^{\prime}$, use the $O\left(m^{3}+m n^{2} \log n\right)$ algorithm from [10], which is the fastest algorithm to compute an MCB without fast matrix multiplication. The algorithm in Figure 1 can also be implemented without fast matrix multiplication. The last phase is the only part which depends on fast matrix multiplication techniques. Instead of using the algorithm in [21] we can use the original approach from de Pina [10]. The running time of this 


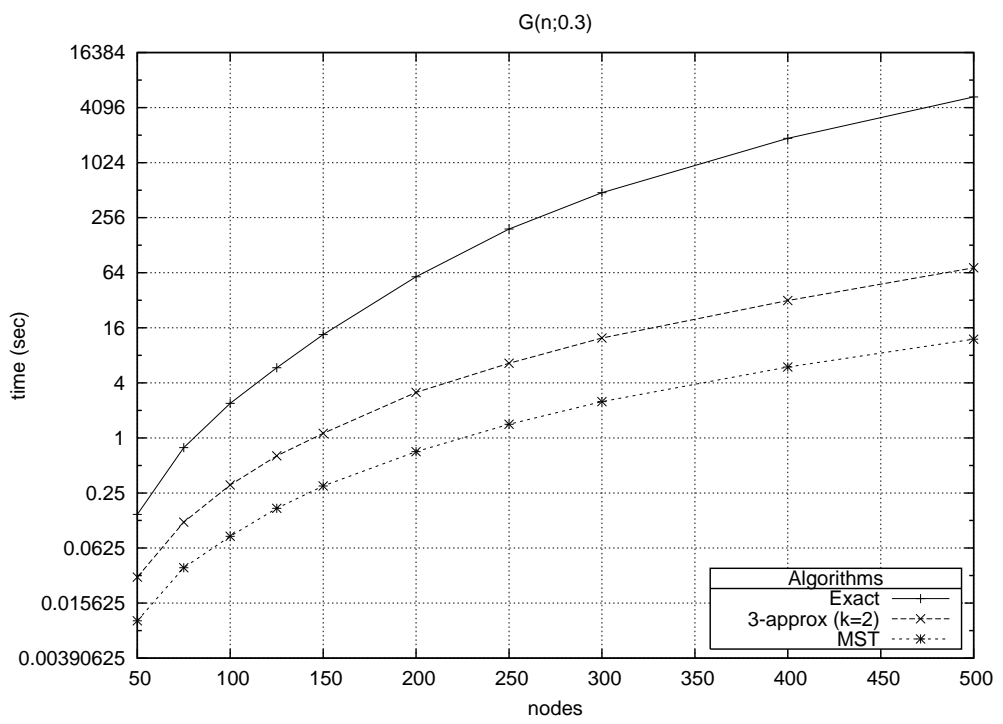

Figure 3: Performance of the algorithm in Figure 2 in practice for $G(n ; 0.3)$ random weighted dense graphs. Three variants are used (a) exact computation for $k=1$, (b) 3 approximation for $k=2$, and (c) $O(n)$ approximation for $k=n$. For $k=n$ the algorithm runs in $O(m n)$ and returns the fundamental basis induced by the minimum spanning tree of the graph. A fundamental basis induced by a spanning tree $T$ is the following cycle basis: for each $e \notin T$ one cycle consisting of $e$ and the unique path in $T$ between its endpoints. We include it here for comparison, since, a spanning tree is the best (sparsest) one can hope for a spanner.

approach is $O\left(m \mu^{2}\right)=O\left(m n^{2+2 / k}\right)$ plus $O\left(\mu\left(k n+k m n^{1 / k}\right)\right)$ for the shortest paths. Thus, we get the following theorem.

Theorem 5.1. The algorithm in Figure 2 computes a $(2 k-1)$-approximate minimum cycle basis, for any integer $k \geq 1$, in an undirected weighted graph without fast matrix multiplication in time $O\left(n^{3+3 / k}\right)$. Similarly, the algorithm in Figure 1 in expected time $O\left(k m n^{1+2 / k}+m n^{2+2 / k}\right)$.

Both our algorithms are $o\left(m^{\omega}\right)$ for sufficiently dense graphs and appropriate values of $k$. Moreover, they are easy to implement efficiently. We performed some preliminary experiments and as it is to be expected, the 3 approximation algorithm is significantly faster than the exact approach since it reduces the problem into a computation in a sparser graph. See Figure 3.

\section{A 2-approximation algorithm}

Another possible way to compute an MCB is to use the greedy algorithm. Sort cycles in nondecreasing order of weight and use Gaussian elimination. The approach works since the sets of linearly independent vectors of a vector space form a matroid. Hence, the greedy algorithm can determine a least weight basis. A graph, however, may have an exponential number of cycles.

Horton [16] defined a set of $O(m n)$ cycles and proved that it contains an MCB. Thus, an MCB can be found by determining the least weight $N$ linearly independent cycles from this set, using Gaussian elimination. We define a set of $O(m \sqrt{n \log n})$ cycles and show that it contains a 2-approximate minimum cycle basis; our set is a subset of Horton's set. Again, the basis is extracted from the set by determining the least weight $N$ linearly independent cycles in it. 
For a vertex $x \in V$ and an edge $e=(u, v) \in E$, let $C[x, e]=\operatorname{SP}(x, u)+e+\operatorname{SP}(v, x)$ be the cycle consisting of the edge $e$ and the shortest paths from $x$ to its endpoints. Horton's collection consists of the cycles $C[x, e]$ for all $x \in V$ and $e \in E$. We use a subset of Horton's collection.

Definition 6.1. For $v, x \in V$ and $S \subset V$, bunch $(v, S)$ consists of all vertices closer to $v$ than to any vertex in $S$ and cluster $(x, S)$ consists of all vertices $v$ with $x \in \operatorname{bunch}(v, S)$.

Lemma 6.1 (Thorup and Zwick [32]). Given a weighted graph $G=(V, E)$ and $0<q<1$, one can compute a set $S \subset V$ of size $O(n q \log n)$ in expected time $O\left(\frac{m \log n}{q}\right)$ such that $\mid$ cluster $(x, S) \mid \leq \frac{4}{q}$ for all $x \in V$.

We take $q=1 / \sqrt{n \log n}$ here and first compute, as given in Lemma 6.1, in expected time $O\left(m \sqrt{n} \log ^{3 / 2} n\right)$ a set $S \subset V$ of $O(\sqrt{n \log n})$ vertices. This ensures that $\operatorname{cluster}(v, S)$ has size $\sqrt{n \log n}$ for all $v \in V$. Also, bunch $(v, S)$ for all $v$ can be computed in expected time $O(m / q)$ [33], which is $O(m \sqrt{n \log n})$. We use two types of cycles:

- the $O(m \sqrt{n \log n})$ cycles $C[s, e]$ for all $s \in S$ and $e \in E$,

- the cycles $C[u, e]$ for each $u \in V$ and $e=(v, w) \in E$ and either $v$ or $w$ in $\operatorname{bunch}(u, S)$. The number of such cycles is $\sum_{u \in V} \sum_{v \in \operatorname{bunch}(u, S)} \operatorname{deg}(v)$. This is the same as $\sum_{v \in V} \operatorname{deg}(v) \cdot|\operatorname{cluster}(v, S)|$, which in turn is at most $\sqrt{n \log n} \sum_{v \in V} \operatorname{deg}(v)=m \sqrt{n \log n}$.

Thus, our collection has $O(m \sqrt{n \log n})$ cycles. We need to show that it contains a 2-approximate cycle basis. Let $B_{1}, \ldots, B_{N}$ be the minimum cycle basis of $G$ determined by Horton's algorithm in order of non-decreasing weight, i.e., $w\left(B_{1}\right) \leq w\left(B_{2}\right) \leq \cdots \leq w\left(B_{N}\right)$.

Lemma 6.2. For all $1 \leq i \leq N$ we have $B_{i}=\sum_{C \in \mathscr{C}_{i}} C$ where $\mathscr{C}_{i}$ is a subset of our collection and each cycle in $\mathscr{C}_{i}$ has cost at most $2 \cdot w\left(B_{i}\right)$.

Proof. Consider any $B_{i}$. If $B_{i}$ belongs to our collection, we set $\mathscr{C}_{i}=\left\{B_{i}\right\}$. Otherwise, $B_{i}=C[u, e]$ where $e=(v, w)$ and neither $v$ nor $w$ is in $\operatorname{bunch}(u, S)$. Let $s \in S$ be the nearest vertex in $S$ to $u$. Then, $w(\operatorname{SP}(s, u)) \leq w(\operatorname{SP}(u, v))$ and $w(\operatorname{SP}(s, u)) \leq w(\operatorname{SP}(u, w))$.

For any edge $f \in B_{i}$, the cycle $C(s, f)$ is in our collection and $B_{i}=\sum_{f \in B_{i}} C(s, f)$ since the paths from $s$ to the endpoints of the edges in $B_{i}$ appear twice in this sum and cancel out. We set $\mathscr{C}_{i}=$ $\left\{C(s, f) \mid f \in B_{i}\right\}$. It remains to show $w(C(s, f)) \leq 2 w\left(B_{i}\right)$ for all $f \in B_{i}$. We distinguish cases.

Assume first that $f \neq e$. Then $f \in \operatorname{SP}(u, v)$ or $f \in \operatorname{SP}(u, w)$. We may assume w.l.o.g. that the former is the case. Then $w(C(s, f)) \leq w(\mathrm{SP}(s, u))+w(\mathrm{SP}(u, v))+w(\mathrm{SP}(v, s))$ since $C(s, f)$ consists of $f$ and the shortest paths from $s$ to the endpoints of $f$ and $w(\operatorname{SP}(v, s)) \leq w(\operatorname{SP}(s, u))+w(\operatorname{SP}(u, v))$ by the triangle inequality. Thus $w(C(s, f)) \leq 2(w(\mathrm{SP}(s, u))+w(\operatorname{SP}(u, v))) \leq 2 w\left(B_{i}\right)$ since $w(\operatorname{SP}(s, u)) \leq$ $w(\mathrm{SP}(u, w))$.

Assume next that $f=e$. Then $w(C(s, f))=w(\operatorname{SP}(s, v))+c(e)+w(\operatorname{SP}(w, s)) \leq w(\operatorname{SP}(s, u))+$ $w(\mathrm{SP}(u, v))+c(e)+w(\mathrm{SP}(s, u))+w(\mathrm{SP}(u, w)) \leq 2 w(\operatorname{SP}(u, v))+c(e)+2 w(\operatorname{SP}(u, w)) \leq 2 w\left(B_{i}\right)$.

Lemma 6.3. The collection constructed above contains $N$ linearly independent cycles $A_{1}, \ldots, A_{N}$ with $w\left(A_{i}\right) \leq 2 \cdot w\left(B_{i}\right)$ for $i=1, \ldots, N$.

Proof. The lemma follows from Lemma 6.2. Assume otherwise and let $j$ be minimal such that $\cup_{i \leq j} \mathscr{C}_{i}$ contains less than $j$ linearly independent vectors with $w\left(A_{i}\right) \leq 2 \cdot w\left(B_{i}\right)$ for $i=1, \ldots, j$. Then $j \geq 1$ and $\cup_{i \leq j-1} \mathscr{C}_{i}$ contains at least $j-1$ linearly independent vectors with $w\left(A_{i}\right) \leq 2 \cdot w\left(B_{i}\right)$ for $i=1, \ldots, j-1$. Also, $\cup_{i \leq j} \mathscr{C}_{i}$ spans $\left\{B_{1}, \ldots, B_{i}\right\}$ and hence contains at least $i$ linearly independent vectors. Thus, it contains a vector $A_{j}$ linearly independent from $\left\{A_{1}, \ldots, A_{j-1}\right\}$. Furthermore, $A_{j} \in \mathscr{C}_{i}$ for some $i \leq j$ and hence $w\left(A_{j}\right) \leq 2 w\left(B_{i}\right) \leq 2 w\left(B_{j}\right)$, a contradiction. 
We sort our collection in non-decreasing order of weight and do Gaussian elimination on their incidence vectors, restricted to the $N$ edges $e_{1}, \ldots, e_{N}$. This determines the least weight $N$ linearly independent cycles in our collection. The time taken for the Gaussian elimination step, which is the most expensive step in our algorithm, is $O\left(m^{\omega} \sqrt{n \log n}\right)$ using fast matrix multiplication.

Theorem 6.4. A 2-approximate $\mathrm{MCB}$ in an undirected graph $G$ with non-negative edge weights can be computed in expected time $O\left(m^{\omega} \sqrt{n \log n}\right)$.

\subsection{Extension to directed graphs}

The above algorithm also holds for directed graphs. See Section 4.4 for definitions. Let $C=\left(e_{1}, \ldots, e_{k}\right)$ be a cycle in a directed graph and let $e_{i}=\left(u_{i}, u_{i+1}\right)$. Then we can write $C=\sum_{i=1}^{k} \operatorname{SP}\left(s, u_{i}\right)+e_{i}+$ $\operatorname{SP}\left(u_{i+1}, s\right)$ where $u_{k+1}=u_{1}$, since $\operatorname{SP}\left(s, u_{i}\right)$ cancels $\operatorname{SP}\left(u_{i}, s\right)$. Note that $\operatorname{SP}(a, b)$ for us here need not be a directed path - it is a shortest path in the underlying undirected graph between $a$ and $b$. However, the incidence vector of this path in the directed graph would contain -1 's corresponding to edges which are traversed in the reverse direction. All the steps in the above construction go through for directed minimum cycle bases too and we have a collection of $O(m \sqrt{n \log n})$ cycles which is a superset of a 2-approximate directed minimum cycle basis.

However, when we do Gaussian elimination, we are no longer over $\mathbb{F}_{2}$ and so the numbers could grow large. So we can no longer claim that the time taken for Gaussian elimination is $O\left(m^{\omega} \sqrt{n \log n}\right)$. But if we choose a prime $p$ uniformly at random from a collection of small primes and do the arithmetic in Gaussian elimination modulo $p$, then our cost remains $O\left(m^{\omega} \sqrt{n \log n}\right)$ and we will show that with high probability we determine the cycles of a 2-approximate MCB.

Arithmetic modulo $p$. The problem with doing arithmetic modulo any number $p$ is that the least weight $N$ linearly independent cycles in our collection could turn out to be linearly dependent modulo $p$. That is, the determinant of the $N \times N$ matrix $M$, defined by incidence vectors of these $N$ cycles, is a multiple of $p$. In that case, our algorithm is not guaranteed to return a 2-approximate minimum cycle basis.

Now we will use the property that all the entries in the matrix $M$ are in $\{-1,0,1\}$, to show a bounded error when $p$ is a prime chosen uniformly at random from a collection $P=\left\{p_{1}, \ldots, p_{N^{2}}\right\}$ of $N^{2}$ distinct primes, where each $p_{i} \geq \sqrt{N}$. It follows from Hadamard's inequality that the absolute value of the determinant of $M$ is at most $N^{N / 2}$, since each of the $N$ rows is a vector in $\{-1,0,1\}^{N}$. Thus, at most $N$ elements of $P$ can be divisors of $\operatorname{det}(M)$. So the probability that a random element of $P$ divides $\operatorname{det}(M)$ is $\leq N / N^{2}=1 / N$. So with probability $1-1 / N$, arithmetic modulo $p$ yields the least weight $N$ linearly independent cycles from the collection of $O(m \sqrt{n \log n})$ cycles.

The value of $\pi(r)$, the number of primes less than $r$, is given by $\frac{r}{6 \log r} \leq \pi(r) \leq \frac{8 r}{\log r}$ [2]. So all the primes $p_{1}, \ldots, p_{N^{2}}$ are $\tilde{O}\left(N^{2}\right)$, and computing them takes $\tilde{O}\left(N^{2}\right)$ time using a sieving algorithm. Arithmetic modulo $p$ ensures that all numbers are $\tilde{O}\left(N^{2}\right)$ and we can assume that arithmetic on $O(\log N)$ bit numbers takes $O(1)$ time. It follows that addition, subtraction and multiplication in $\mathbb{Z}_{p}$ can be implemented in unit time since $p$ is $\tilde{O}\left(N^{2}\right)$. However, we also need to implement division efficiently. Once $p$ is chosen, we compute the multiplicative inverses of all elements in $\mathbb{Z}_{p}^{*}$ by the extended Euclid's gcd algorithm by solving $a x=1(\bmod p)$ for each $a \in \mathbb{Z}_{p}^{*}$. This takes time $O(\log p)$ for each element and hence $O(p \log p)=\tilde{O}\left(N^{2}\right)$ for all the elements. Thus, we have shown the following theorem.

Theorem 6.5. A 2-approximate minimum cycle basis can be computed with high probability in expected time $O\left(m^{\omega} \sqrt{n \log n}\right)$ in an directed graph $G$ with $n$ vertices, $m$ edges and non-negative edge weights. 


\section{Conclusions and open problems}

In this paper we design faster algorithms for computing approximate minimum cycle basis of undirected graphs. To the best of our knowledge it is the first time that sparse cycle bases with a guarantee are computed in $o\left(m^{\omega}\right)$ time. The importance of this result follows from the fact that minimum cycle bases are usually used to form linear systems which then have to be solved. Thus, the MCB computation should not dominate the running time. Our techniques extend also to the directed version of the minimum cycle basis problem in which the base field is $\mathbb{Q}$ instead of $\mathbb{F}_{2}$. We present very fast approximate algorithms for this version as well.

One of the most important open questions in this area is to decouple the (approximate) MCB computation from the null space basis. We have partially solved this by computing all but $O\left(n^{1+1 / k}\right)$ cycles without using a null space basis. Another related question to this is whether we can reach an $o\left(m^{\omega}\right)$ running time when computing exactly the minimum cycle basis or is there an $\Omega\left(m^{\omega}\right)$ lower bound?

\section{References}

[1] Ingo Althöfer, Gautam Das, David Dobkin, Deborah Joseph, and José Soares. On sparse spanners of weighted graphs. Discrete Comput. Geom., 9(1):81-100, 1993.

[2] T. M. Apostol. Introduction to Analytic Number Theory. Springer-Verlag, 1997.

[3] Surender Baswana and Sandeep Sen. Approximate distance oracles for unweighted graphs in expected o(n2) time. ACM Trans. Algorithms, 2(4):557-577, 2006.

[4] F. Berger, P. Gritzmann, and S. de Vries. Minimum cycle basis for network graphs. Algorithmica, 40(1):51-62, 2004.

[5] Paul Callahan and Rao Kosaraju. A decomposition of multidimensional point sets with applications to $k$-nearest-neighbors and $n$-body potential field. Journal of the ACM, 42(1):67-90, 1995.

[6] A. C. Cassell, J. C. Henderson, and K. Ramachandran. Cycle bases of minimal measure for the structural analysis of skeletal structures by the flexibility method. In Proc. Royal Society of London Series A, volume 350, pages 61-70, 1976.

[7] D. Coppersmith and S. Winograd. Matrix multiplications via arithmetic progressions. Journal of Symb. Comput., 9:251-280, 1990.

[8] Thomas H. Cormen, Charles E. Leiserson, and Ronald L. Rivest. Introduction to Algorithms. The MIT Press and McGraw-Hill Book Company, 1989.

[9] Kreisbasenbibliothek CyBaL. http: //www-m9.ma.tum.de/dm/cycles / cybal, 2004.

[10] J.C. de Pina. Applications of Shortest Path Methods. PhD thesis, University of Amsterdam, Netherlands, 1995.

[11] Petra Manuela Gleiss. Short Cycles, Minimum Cycle Bases of Graphs from Chemistry and Biochemistry. PhD thesis, Fakultät Für Naturwissenschaften und Mathematik der Universität Wien, 2001. 
[12] A. Golynski and J. D. Horton. A polynomial time algorithm to find the minimum cycle basis of a regular matroid. In 8th Scandinavian Workshop on Algorithm Theory, 2002.

[13] Ramesh Hariharan, Telikepalli Kavitha, and Kurt Mehlhorn. A faster deterministic algorithm for minimum cycle basis in directed graphs. In ICALP 2006, 33rd International Colloquium on Automata, Languages and Programming, volume 4051 of LNCS, pages 250-261, 2006.

[14] David Hartvigsen and Russell Mardon. The all-pairs min cut problem and the minimum cycle basis problem on planar graphs. Journal of Discrete Mathematics, 7(3):403-418, 1994.

[15] J. Hopcroft and R. Tarjan. Efficient planarity testing. Journal of the ACM, 21:549-568, 1974.

[16] J. D. Horton. A polynomial-time algorithm to find a shortest cycle basis of a graph. SIAM Journal of Computing, 16:359-366, 1987.

[17] M. Huber. Implementation of algorithms for sparse cycle bases of graphs. Technical report, Technische Universität München, 2002. http://www-m9.ma.tum.de/dm/cycles/ mhuber.

[18] Telikepalli Kavitha. An $\tilde{O}\left(m^{2} n\right)$ randomized algorithm to compute a minimum cycle basis of a directed graph. In Proceedings of ICALP, LNCS 3580, pages 273-284, 2005.

[19] Telikepalli Kavitha and Kurt Mehlhorn. Algorithms to compute minimum cycle basis in directed graphs. Theor. Comp. Sys., 40(4):485-505, 2007.

[20] Telikepalli Kavitha, Kurt Mehlhorn, and Dimitrios Michail. New approximation algorithms for minimum cycle bases of graphs. In STACS 2007, 24th Annual Symposium on Theoretical Aspects of Computer Science, volume 4393 of LNCS, pages 512-523, 2007.

[21] Telikepalli Kavitha, Kurt Mehlhorn, Dimitrios Michail, and Katarzyna E. Paluch. An $\tilde{O}\left(m^{2} n\right)$ algorithm for minimum cycle basis of graphs. Algorithmica, 2007. DOI: 10.1007/s00453-0079064-z.

[22] J. Mark Keil and Carl A. Gutwin. Classes of graphs which approximate the complete euclidean graph. Discrete Computational Geometry, 7:13-28, 1992.

[23] Josef Leydold and Peter F. Stadler. Minimal cycle bases of outerplanar graphs. Electron. J. of Combinatorics, 5:1-14, 1998.

[24] Christian Liebchen and Romeo Rizzi. A greedy approach to compute a minimum cycle basis of a directed graph. Inf. Process. Lett., 94(3):107-112, 2005.

[25] Christian Liebchen and Romeo Rizzi. Classes of cycle bases. Discrete Appl. Math., 155(3):337$355,2007$.

[26] Kurt Mehlhorn and Dimitrios Michail. Implementing minimum cycle basis algorithms. J. Exp. Algorithmics, 11:1-14, 2006.

[27] Romeo Rizzi. Minimum weakly fundamental cycle bases are hard to find. Algorithmica, 2007. DOI: 10.1007/s00453-007-9112-8. 
[28] Liam Roditty, Mikkel Thorup, and Uri Zwick. Deterministic constructions of approximate distance oracles and spanners. In Proceedings of the 32nd International Colloquium in Automata, Languages and Programming, LNCS volume 3580, pages 261-272, 2005.

[29] G. F. Stepanec. Basis systems of vector cycles with extremal properties in graphs. Uspekhi Mat. Nauk, 19:171-175, 1964.

[30] M. N. S. Swamy and K. Thulasiraman. Graphs, Networks, and Algorithms. John Wiley \& Sons, New York, 1981.

[31] Geetika Tewari, Craig Gotsman, and Steven J. Gortler. Meshing genus-1 point clouds using discrete one-forms. Comput. Graph., 30(6):917-926, 2006.

[32] Mikkel Thorup and Uri Zwick. Compact routing schemes. In Proceedings of 13th ACM Symposium on Parallel Algorithms and Architecture, pages 1-10, 2001.

[33] Mikkel Thorup and Uri Zwick. Approximate distance oracles. J. ACM, 52(1):1-24, 2005. 